\title{
Optipris : A Constant Pressure Air Intake for a Mach Number up to 0.3
}

\author{
Pierre-Louis Schmitt, Anthony Haroutunian † Patrick Haas $\ddagger$ Michel Perraudin § \\ CMEFE - Aerodynamics Group \\ University of Applied Sciences Western Switzerland HES-SO, EIG, Geneva, CH 1202, Switzerland
}

25th AIAA Applied Aerodynamics Conference, 25 - 28 June 2007, Miami, Florida.

Paper AIAA-2007-3923

\begin{abstract}
This study takes place within the framework of the Optipris project whose goal is to develop high-performance air intakes. Most of the time, air intakes are located in regions of negative pressure coefficients. In these cases, a flow regulation is necessary for any application for which the mass flow is prevalent. For example in the case of air conditioning systems. An intake offering a constant pressure at the inlet offers a great advantage: the fresh air mass flow is not influenced by the vehicule speed.

In this project, a fully immerged intake yielding a constant mass flow is developed and validated for the incompressible domain. The development process is based on CFD simulations. Physical models are also built and tested in a subsonic wind tunnel to validate the simulation models.

The intake design parameters are evaluated for several applications and pressure coefficients. Similarity criteria are developed. Then, the sensitive design parameters are determined. A design law is obtained from which it is possible to define a constant pressure air intake for given flow rates and pressure coefficients. These data are validated for a range of pressure coefficients from 0 to $\mathbf{- 0 . 3}$ and for the incompressible flow regime.
\end{abstract}

\footnotetext{
*Research Assistant, CMEFE/IMEC/EIG/HES-SO, Rue de la Prairie 4, CH 1202 Geneva, http://www.cmefe.ch, graduate student at Ecole des Arts et Metiers, Paris, AIAA Student Member

${ }^{\dagger}$ Research Assistant, CMEFE/IMEC/EIG/HES-SO, Rue de la Prairie 4, CH 1202 Geneva, http://www.cmefe.ch, AIAA Student Member

${ }^{\ddagger}$ Professor, CMEFE/IMEC/EIG/HES-SO, Rue de la Prairie 4, CH 1202 Geneva, http://www.cmefe.ch, AIAA Senior Member

${ }^{\S}$ Head of CMEFE, CMEFE/IMEC/EIG/HES-SO, Rue de la Prairie 4, CH 1202 Geneva, http://www.cmefe.ch, AIAA Member
} 

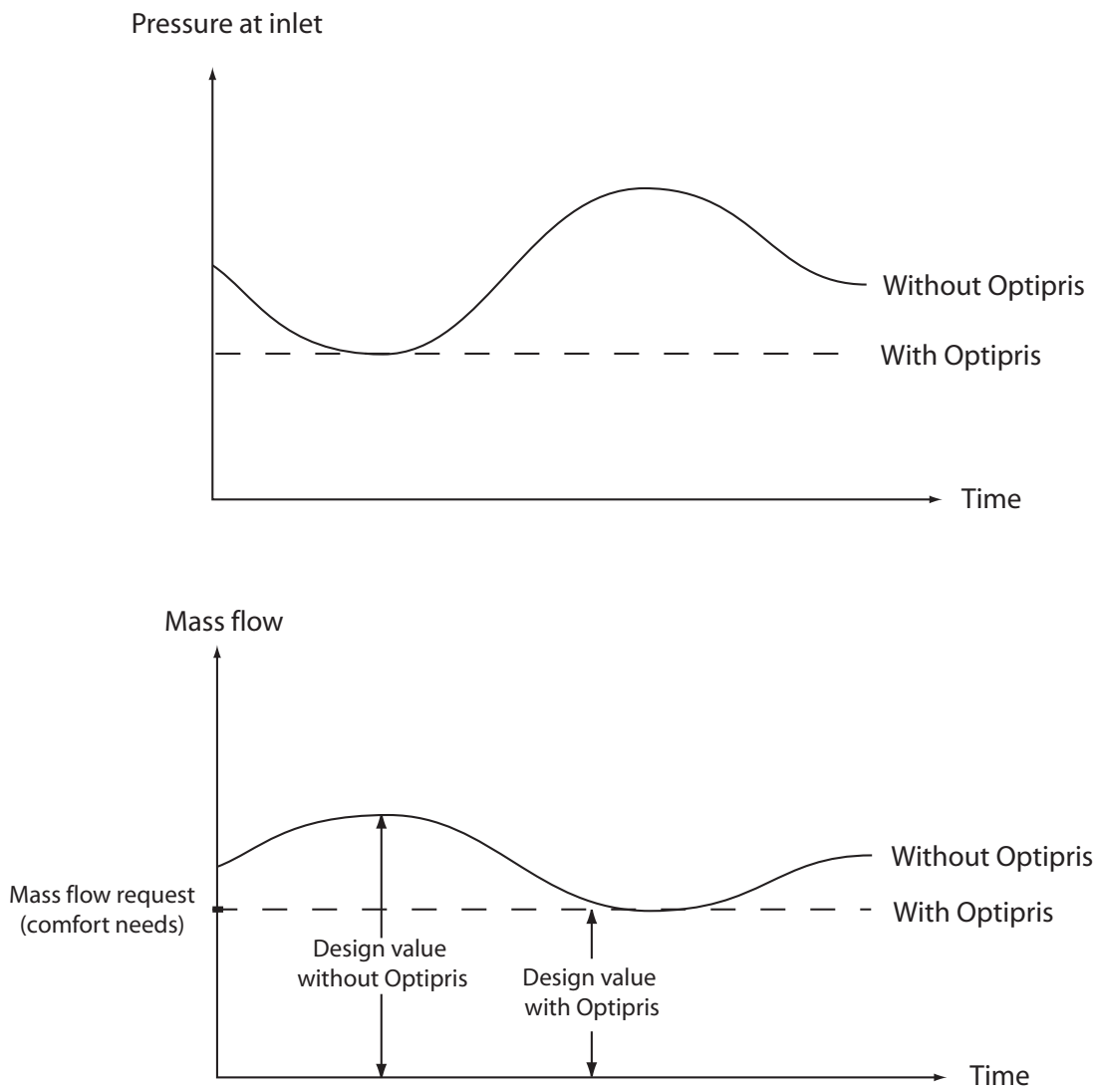

Figure 1. Variation of the mass flow at the inlet during a journey for an HVAC inlet located in a region of non-zero pressure coefficient.

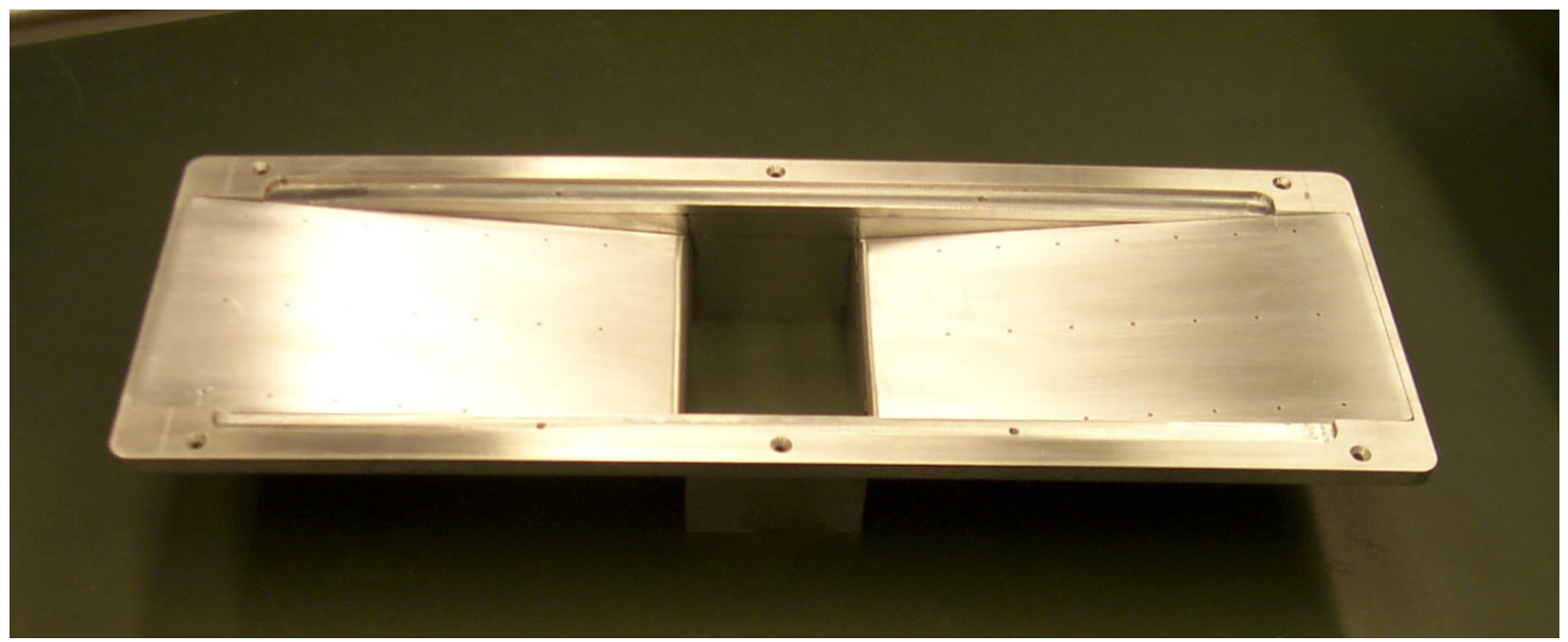

Figure 2. Rectangular diffusor Optipris model (here without horizontal plate). 


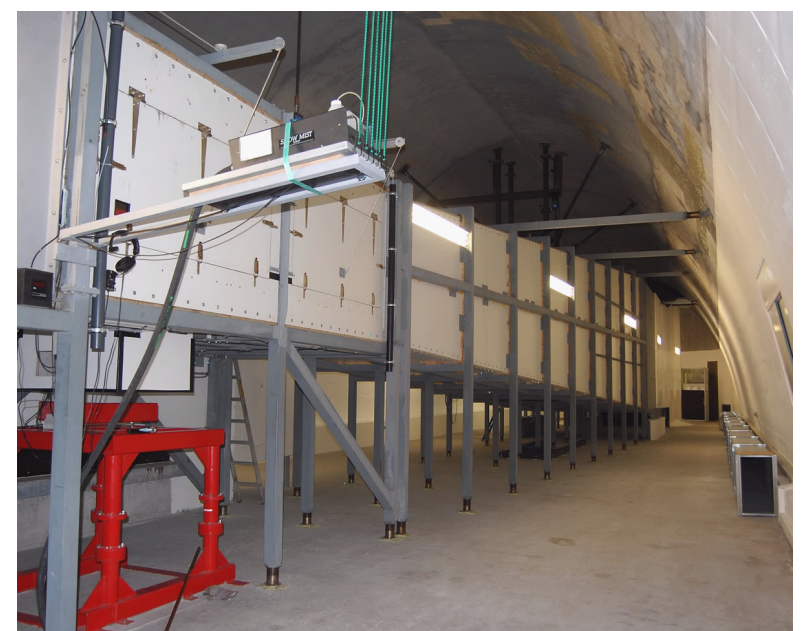

(a)

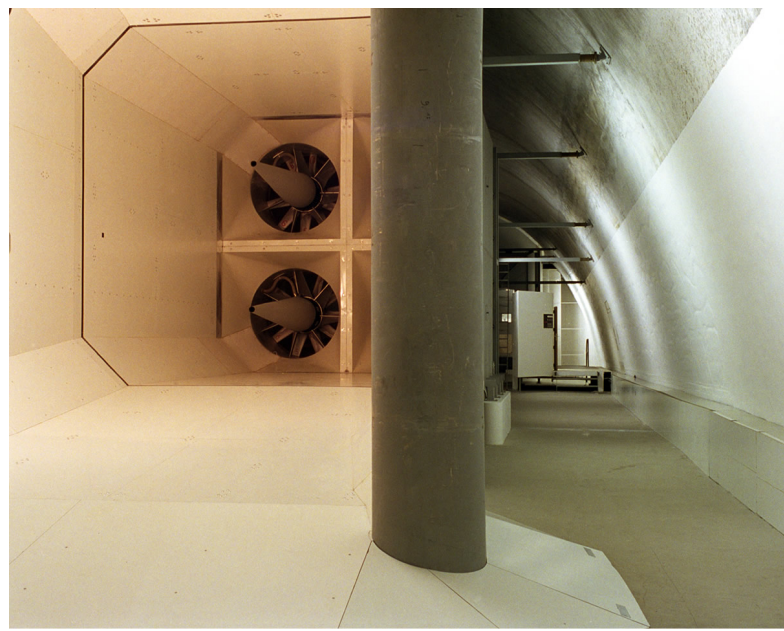

(c)

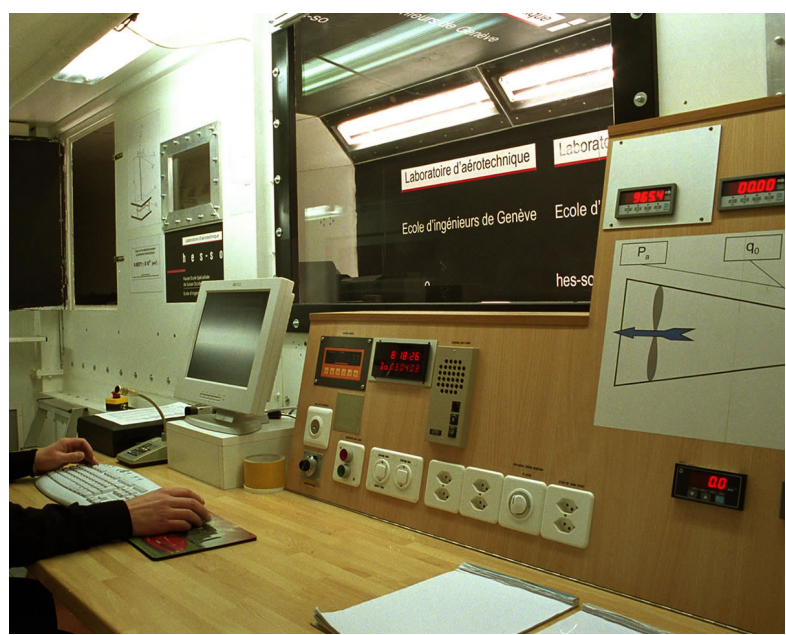

(b)

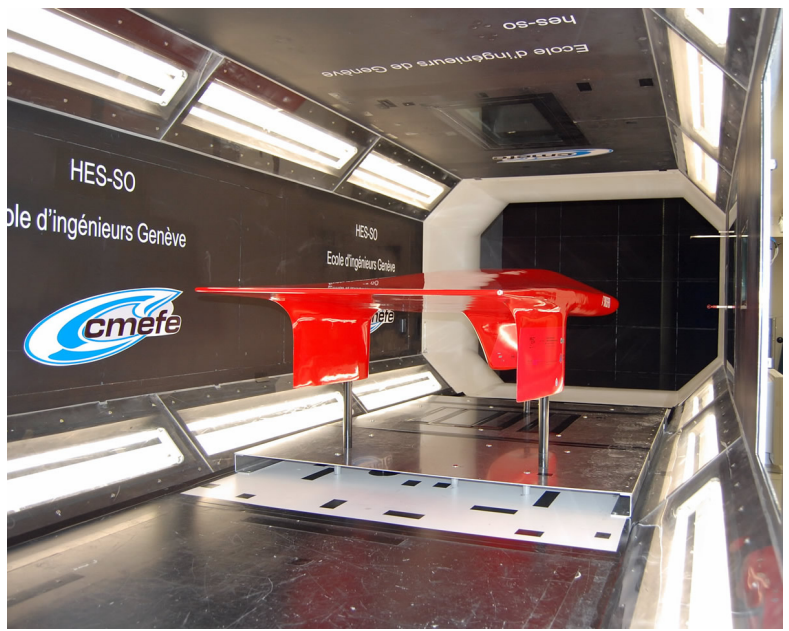

(d)

Figure 3. Some views of the CMEFE large subsonic wind tunnel : (a)diffusor; (b)control room; (c)blowers; (d)2.0 m $\mathrm{x}$ $1.5 \mathrm{~m}$ test section. 